\title{
Antibody-mediated inhibition of HIV-1 elicited by HIV-I DNA priming and boosting with heterologous HIV-1 recombinant MVA in healthy Tanzanian adults
}

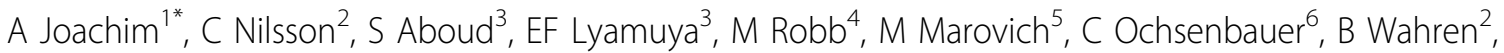 \\ E Sandström ${ }^{7}, \mathrm{G}$ Biberfeld ${ }^{2}, \mathrm{G}$ Ferrari ${ }^{8}$, VR Polonis ${ }^{5}$
}

From AIDS Vaccine 2012

Boston, MA, USA. 9-12 September 2012

\section{Background}

We evaluated HIV antibody $(\mathrm{Ab})$ responses elicited by immunization, in a phase I/II placebo-controlled double blind trial using multiclade, multigene HIV-1-DNA prime boosted with HIV-MVA conducted among healthy volunteers in Tanzania (HIVIS03).

\section{Methods}

Sixty HIV-uninfected volunteers, randomized into groups of 20 received placebo or $1 \mathrm{mg}$ HIV-DNA intradermally (id) or $3.8 \mathrm{mg}$ intramuscularly (im). DNA plasmids containing HIV-1 gp160 subtypes A, B, C; rev B; p17/p24 gag $\mathrm{A}, \mathrm{B}$ and RTmut $\mathrm{B}$ were given at months 0,1 and 3 using a needle-free Biojector device. HIV-MVA expressing CRF01_AE HIV-1 env, gag, pol was administered im by needle at months 9 and 21. Sera were tested at baseline, two months post-first and four weeks post-second HIV-MVA boosting. HIV Ab responses were tested using pseudoviruses and TZM-bl cells as well as luciferaseexpressing infectious molecular clones (IMC-LucR) in PBMC-based assays. ADCC responses were tested using the flow cytometry GranToxiLux-based assay.

\section{Results}

Neutralizing Ab activity was demonstrated only in the PBMC assay, and after the second MVA boost in 24 $(83 \%)$ of 29 vaccinees against the clade CRF01_AE CM235 IMC and in 21 (72\%) of 29 vaccinees against

'Muhimbili University of Health and Allied Sciences, Dar es Salaam, Tanzania, United Republic of

Full list of author information is available at the end of the article clade B SF162-IMC. NK cell depletion from PBMC targets resulted in a significant loss of HIV inhibition by vaccinee sera, indicating a role of Ab-mediated Fcy-receptor function. Vaccine-induced ADCC responses were detected in $21(75 \%)$ of 28 vaccinees after the second HIV-MVA boost. ADCC Ab titers did not differ significantly between id- (median 840, range 300-5400) and imprimed (median 880 , range $400-3600)$ vaccinees $(\mathrm{p}=0.45)$.

\section{Conclusion}

HIV-DNA priming followed by two HIV-MVA boosts elicited HIV-specific inhibitory and/or ADCC-mediating antibody responses in a high proportion of Tanzanian adults.

\section{Author details}

'Muhimbili University of Health and Allied Sciences, Dar es Salaam, Tanzania, United Republic of. ${ }^{2}$ Karolinska Institutet / Institute for Infectious Disease Control (SMI), Stockholm, Sweden. ${ }^{3}$ Muhimbili University of Health and Allied Sciences (MUHAS), Dar es Salaam, Tanzania, United Republic of. ${ }^{4}$ The Henry M. Jackson Foundation, Rockville, MD, USA. ${ }^{5}$ Walter Reed Army Institute of Research (WRAIR), Rockville, MD, USA. 'Department of Medicine, University of Alabama at Birmingham, Birmingham, AL, USA. Venhälsan, Karolinska Institutet (KI) at Södersjukhuset, Stockholm, Sweden. ${ }^{8}$ Department of Surgery, Duke University Medical Center, Durham, NC, USA.

Published: 13 September 2012

doi:10.1186/1742-4690-9-S2-053

Cite this article as: Joachim et al: Antibody-mediated inhibition of HIV-1 elicited by HIV-I DNA priming and boosting with heterologous HIV-1 recombinant MVA in healthy Tanzanian adults. Retrovirology 20129 (Suppl 2):053. 